\title{
【研究简报】
}

\section{南极乔治王岛第三纪火山岩 $\mathrm{Sr}, \mathrm{Nd}$ 和 $\mathrm{Pb}$ 同位素研究}

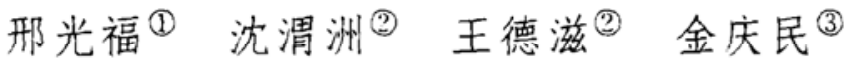

(1)中国地质科学院南京地质矿产研究所, 南京 210016;

(2)南京大学地球科学系, 南京 210093; (3)极地科学研究发展基金会, 南京 210016)

\section{关钺词 Sr, Nd, Pb 同位軎 第三纪火山岩 乔治王岛 南极}

\section{1 地质背景}

乔治王岛是南极南设得兰群岛中最大的岛屿, 面积约 $1200 \mathrm{~km}^{2}$, 大部分地区长年被冰雪 覆盖. 全岛广泛分布第三纪火山岩, 也零星出露相应侵人岩及晚中生代和第四纪火山岩. 南设 得兰群岛是一发育于硅铝质基底之上的、时代为侏罗纪-第三纪的陆缘火岩弧, 其岩浆活动受 太平洋板块向南极大陆板块的俯冲所制约; 它曾是南极大陆西部濒太平洋边缘的一部分, 直到 约 $2 \mathrm{Ma}$ 前, 在俯冲作用停止和边缘盆地布兰斯菲尔德海峡张开后才从南极半岛西北部分离出 来 ${ }^{[1]}$ (图 1).

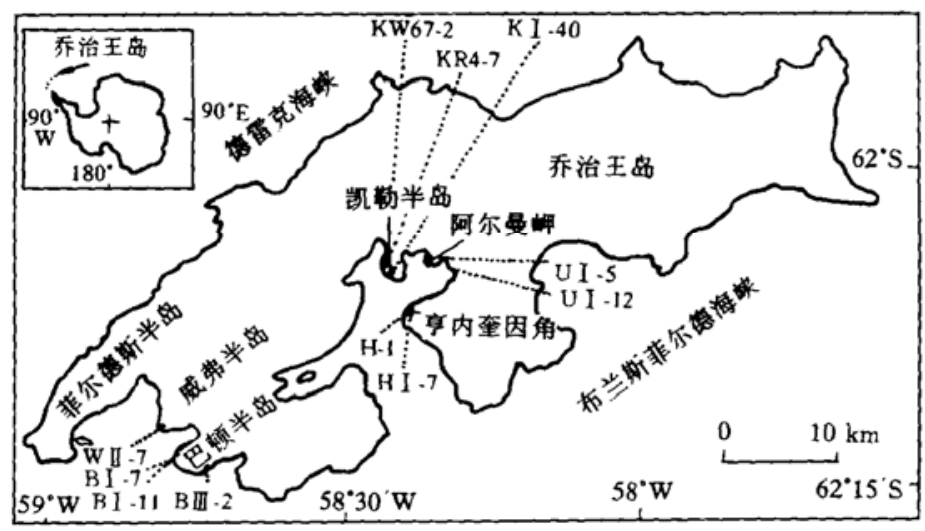

图 1 研究区地理位置与样品分布

乔治王岛第三纪火山岩以高铝玄武岩、玄武安山岩和安山岩为主, 英安岩少量; 岩石系列 在早期主要为拉斑系列, 中晚期过渡为典型的钻碱性系列; 它们的微量元素特征为富 LILE, 在 球粒陨石标准化的稀土元素配分图上明显地富 LREE 和负 $\mathrm{Ce}$ 异常 ${ }^{[2 \sim 5]}$. 以前对乔治王岛火 山岩的同位素研究仅限于 $\mathrm{Sr}$ 同位素,近年来才涉及 $\mathrm{Nd}$ 和 $\mathrm{Pb}$ 同位素.

\section{2 样品与分析方法}

在薄片观察和初步岩石化学研究的基础上, 选择新鲜的或蚀变轻微并经 $\mathrm{HCl}$ 溶液处理过 
的全岩样品作同位素测试. $\mathrm{Sr}, \mathrm{Pb}$ 同位素测试在中国地质科学院宜昌地质矿产研究所同位素 实验室完成, 仪器为 MAT-261 质谱计; 空白本底 $\mathrm{Rb}, \mathrm{Sr}$ 为 $2 \times 10^{10} \mathrm{~g}, \mathrm{~Pb}$ 为 $2 \times 10^{9} \mathrm{~g}$; 分析误 差 $\left({ }^{87} \mathrm{Sr} /{ }^{86} \mathrm{Sr}\right)$ 为 $0.01 \% \sim 0.005 \%, \mathrm{~Pb}$ 同位素比值为 $0.1 \% \sim 0.08 \% . \mathrm{Nd}$ 同位素测试在中国 科学院地质研究所同位素实验室完成, 仪器为 VG-354 质谱计, 仪器对 La Jolla Vd 同位素标准 的测定结果为 ${ }^{1+3} \mathrm{Nd} / /^{1+4} \mathrm{Nd}=0.511858 \pm 0.000010,{ }^{1+5} \mathrm{Nd} /{ }^{1+4} \mathrm{Nd}=0.348380 \pm 0.000017, \mathrm{Nd}$ 同位素质量歧视校正采用 ${ }^{142} \mathrm{Nd} /{ }^{14+} \mathrm{Nd}=1.14169$ 和 ${ }^{1+6} \mathrm{Nd} /{ }^{1+4} \mathrm{Nd}=0.7219$ 同时校正; 空白本 底 $\mathrm{Sm}$ 为 $(1.1 \sim 3.3) \times 101$ " g, Nd 为 $(1.0 \sim 1.7) \times 10{ }^{11} \mathrm{~g}$; 分析误差 ${ }^{1+3} \mathrm{Nd} /{ }^{1+4} \mathrm{Nd}$ 为 $0.001 \%$ $\sim 0.0005 \%$. 结果列于表 1 .

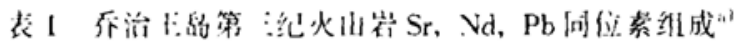

\begin{tabular}{|c|c|c|c|c|c|c|c|c|c|c|}
\hline 样号 & 岁蚆 & $\begin{array}{l}\text { 年䜌 } \\
\text { / Ma }\end{array}$ & ${ }^{n \gamma} \mathrm{Kb},{ }^{* t} \mathrm{Sr}{ }^{n+} \mathrm{Sr} /{ }^{* 6} \mathrm{Sr}$ & $I_{4}$ & ${ }^{147} \operatorname{Sin}^{1+14} / \mathrm{Vd}$ & ${ }^{142} \mathrm{Nd}{ }^{144} \mathrm{Nd}$ & $\varepsilon v(t)$ & 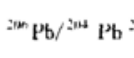 & ${ }^{117} \mathrm{P} b /{ }^{344} \mathrm{~Pb}$ & ${ }^{112} \mathrm{~Pb} /=314 \mathrm{~Pb}$ \\
\hline $\mathrm{kl}+0$ & 玄武安的䍜 & 49.2 & $0 . \overline{047480.70376}$ & 0.70373 & 0.1325 & 0.51962 & 6.72 & 18.515 & 15.568 & 38.301 \\
\hline KW $67-2$ & 吉武落山岁 & 48.75 & $0.02324 \quad 0.703 .349$ & 0.70 .347 & 0.1288 & 1). 512872 & 4.99 & 18.465 & 15.555 & 38.206 \\
\hline $\mathrm{KR} 4.7$ & 去武发咶符 & 48.75 & $0.05297 \quad 0.70394$ & 0.70390 & 0.1350 & 0.512921 & 5.91 & 18.201 & 15.500 & $38.0+1$ \\
\hline U1-5 & 公武策山䇣 & 38.7 & $0.066071 \quad 0.701348$ & $0.703+5$ & $0.12 \times 3$ & 0.512411 & 5.67 & $18.4+2$ & 15.570 & 38.288 \\
\hline 21.12 & 胜安岩 & 40.2 & 0.240320 .70364 & 0.70350 & 0.1342 & 0.5129 .31 & 6.03 & 18.444 & 15.561 & 38.231 \\
\hline $\mathrm{HI}-1$ & 安消岸 & 32.8 & $0.07878 \quad 0.70390$ & 0.70386 & 0.1386 & 0.512866 & 4.69 & 18.494 & 15.570 & 38.308 \\
\hline $\mathrm{HI} \cdot 7$ & 公武安山宸 & 49.36 & 0.233830 .70 .380 & $0.7036+$ & 0.1329 & 0.512937 & 6.23 & 18.368 & 15.564 & 38.228 \\
\hline$B 1-7$ & 离铝公武岩 & 55.9 & $0.03458 \quad 0.70378$ & 0.70375 & 0.1366 & 0.512909 & 5.71 & 18.145 & 15.550 & 38.223 \\
\hline BI-11 & 高铝公武岩 & 55.9 & 0.051330 .70396 & 0.70392 & 0.1416 & 0.512901 & 5.52 & 17.776 & 15.514 & 37.868 \\
\hline B 国 -2 & 安山项政故崖 & 38.5 & 0.126710 .70382 & 0.70375 & 0.1300 & 0.512857 & 4.59 & 18.510 & 15.571 & 38.292 \\
\hline W II -7 & 离铝公武岩 & 31.54 & 0.071710 .70394 & 0.70391 & 0.1538 & 0.512780 & 3.02 & 18.098 & 15.521 & 38.205 \\
\hline
\end{tabular}

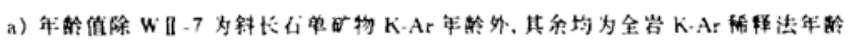

\section{3 结果与讨论}

\section{$3.1 \mathrm{Sr}$ 和 Nd 同位素组成}

由表 1 可见, 乔治王岛第三纪火山岩 $I_{\mathrm{Sr}}=0.70345 \sim 0.70392$, 这与前人报道的结果 $(0.70310 \sim 0.70389)^{[2.4 \sim 7]}$ 基本一致; $\varepsilon_{\mathrm{Nd}}(t)=3.0 \sim 6.7$, 亦与前人的结果 $(2.8 \sim 7.8)^{[4.5 .7]}$ 一致. 值得注意的是, $I_{\mathrm{Sr}}$ 和 $\varepsilon_{\mathrm{Nd}}(t)$ 都有一定的变化范围.

在火山岩的 $\varepsilon_{\mathrm{Nd}}(t)-I_{\mathrm{Sr}}$ 相关图上(图 2), 乔治王岛火山岩投影点均落在地幔系列范围内, 且投影点较集中, 不同于受到海水蚀变影响的马里亚纳和南桑威奇火山岩及受到陆壳物质混 染的小安的列斯和巽他弧火山岩的投影点那样明显偏离地幔系列. 由此可知, 乔治王岛第三 纪火山岩起源于地幔, 岩浆形成与喷发过程中没有受到地壳混染或海水的明显影响. 火山岩 的 Sr 和 Nd 同位素组成变化主要反映其地幔源区同位素组成的不均一. 这种不均一可能是 地幔源区早已存在的,也可能主要是由近期俯冲活动造成的.

\section{$3.2 \mathrm{~Pb}$ 同位素组成}

在表 1 中, 乔治王岛火山岩的 $\mathrm{Pb}$ 同位素组成变化也较明显, 其中 ${ }^{206} \mathrm{~Pb} /{ }^{204} \mathrm{~Pb}=17.776 \sim$ $18.515,{ }^{207} \mathrm{~Pb} /{ }^{204} \mathrm{~Pb}=15.506 \sim 15.571,{ }^{208} \mathrm{~Pb} /{ }^{204} \mathrm{~Pb}=37.868 \sim 38.308$, 这与前人报道的有关 结果也大致类似 ${ }^{[4.5 .7]}$. 在 $\mathrm{Pb}-\mathrm{Pb}$ 同位素相关图上(图 3), 乔治王岛火山岩投影点全部位于北 半球 $\mathrm{Pb}$ 参考线 (NHRI.) $)^{[12]}$ 之上的印度洋 MORB 范围内, 除个别点外明显偏离太平洋和大西 洋 MORB 范围, 反映其地幔源区富放射性成因的 ${ }^{207} \mathrm{~Pb}$ 和 ${ }^{2118} \mathrm{~Pb}$. 这种地幔源区异常地富放射 性成因铅的现象以南半球大洋地幔, 特别是中印度洋脊(CIR) 地幔最为典型, 被命名为南半球 


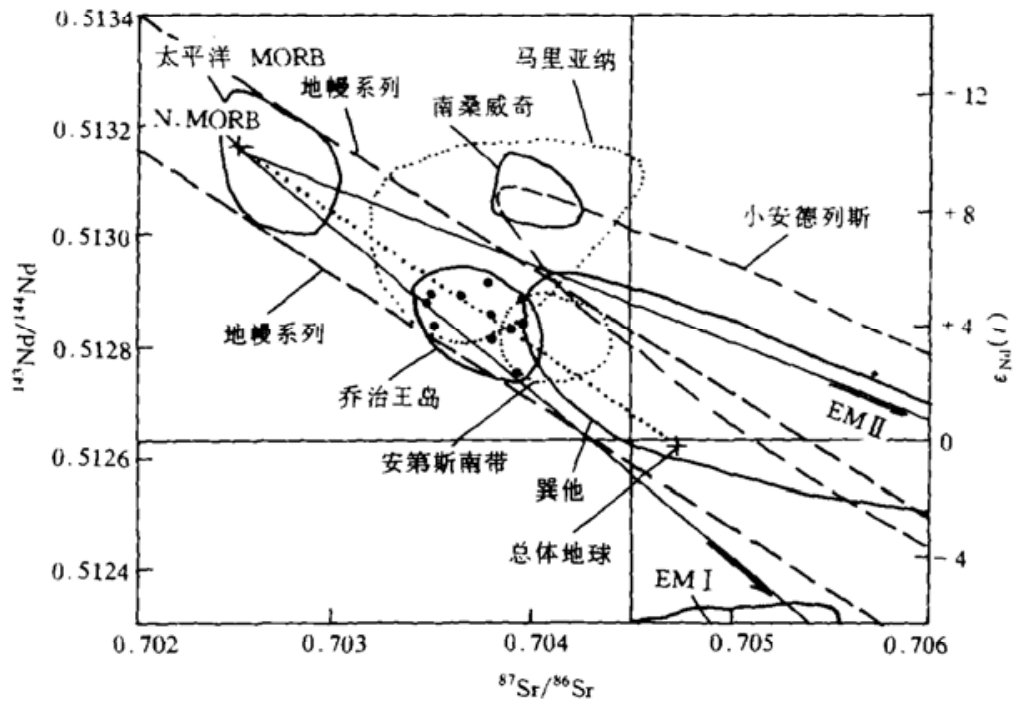

图 2 乔治王岛火山岩 $\varepsilon_{\mathrm{Nd}}(t)-I_{\mathrm{Sr}}$ 相关图

Dupal 异常 $\left(\Delta 8 / 4 \mathrm{~Pb}>60,{ }^{87}\right.$ $\left.\mathrm{Sr} /{ }^{86} \mathrm{Sr}>0.705\right)$, 并认为这是起 源于长期存在的深部地幔 (如核) 幔边界层 $)^{[12,16]}$. 已知菲律宾息 弧火山岩具有类 Dupal 异常 ${ }^{[14]}$, 其形成归因于它们的地幔源区为 由南半球 Dupal 异常带迁移过来 的古老地幔 ${ }^{[17]}$. 虽然乔治王岛 火山岩在图 3(b) 上具有与 CIR MORB 和菲律宾岛弧火山岩类似 的 $\mathrm{Pb}$ 同位素组成, 显示出一定的 类 Dupal 异常特征, 但在图 3(a) 上则表现出整体上比后两者更高 的 ${ }^{207} \mathrm{~Pb} /{ }^{204} \mathrm{~Pb}$, 因而它们的源区 不大可能是后者那样的古老地 幔. 同样由图 3(a)也可看出, 乔 治王岛第三纪火山岩的 $\mathrm{Pb}$ 同位 素组成也不可能经由 CIR 型地幔 与大洋沉积物的直接混合而获 得, 这与它们的低 $I_{\mathrm{Sr}}$ 和高 $\varepsilon_{\mathrm{Nd}}(t)$ 值是一致的.
(背景值引自文裁 $[8 \sim 11]$ )
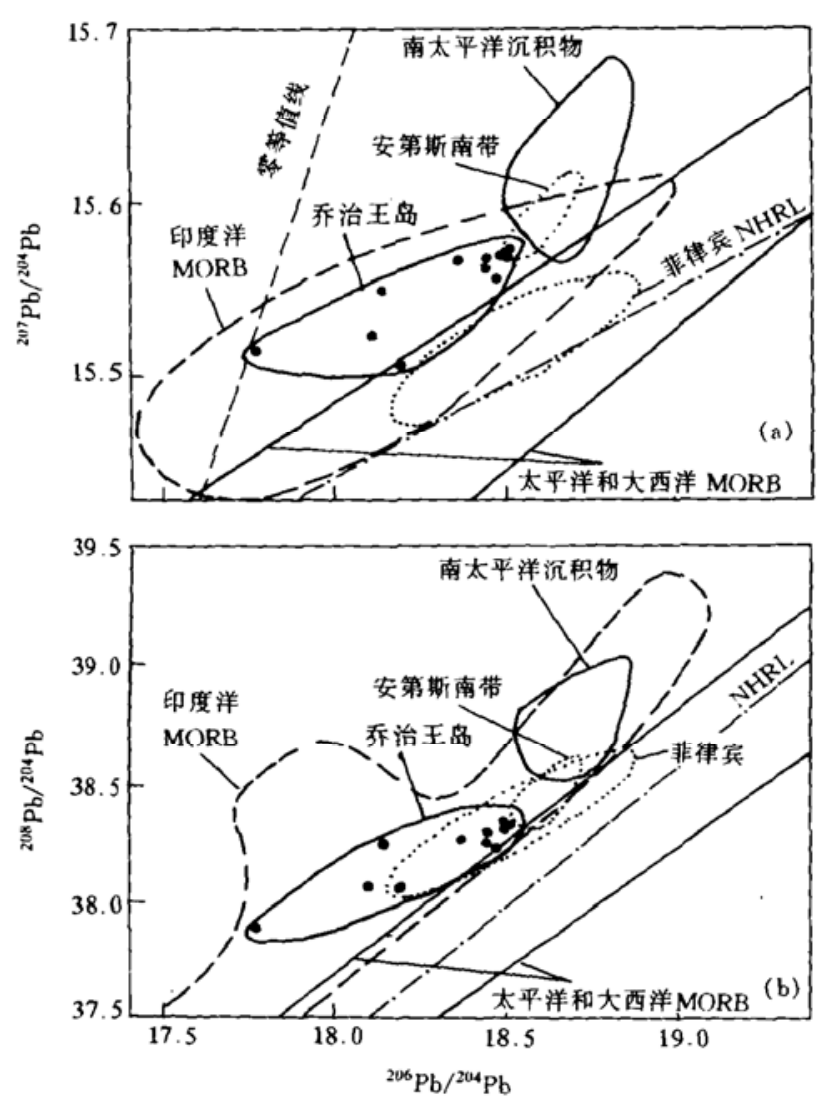

图 3 乔治王岛火山岩 $\mathrm{Pb}$ - $\mathrm{Pb}$ 同位素相关图 (背景值引自文献[12１5]) 


\section{3 源区性质讨论}

南极半岛地区(包括南设得兰群岛)最古老的基底岩石为志留系 ${ }^{[18]}$, 且至少远至 $65^{\circ} \mathrm{S}$. 该 地区既不存在 $U$ 和 $T h$ 富集的上地壳. 也不存在 $U$ 亏损的古老变质基底 ${ }^{[19]}$. 如此较年轻且成 熟度低的陆壳不大可能发生拆沉作用而改变下伏地幔的同位素组成. 另一方面, 南极半岛北 部俯冲板块之下的浅部软流圈地幔是亏损地幔, 不存在类 Dupal 异常 (由其派生的火山岩多数 $\triangle 8 / 4 \mathrm{~Pb}<0)$, 却有一定的 HIMU 特征, 俯冲带之下也不存在地幔柱 ${ }^{[20]}$. 此外, 如上所述, 乔 治王岛第三纪火山岩普遍富 LREE 和 LILE, 说明其地幔源区经历了一定程度的富集, 而它们 的负 $\mathrm{Ce}$ 异常则表明这种富集与俯冲活动有关. 基于以上认识, 我们认为乔治王岛第三纪火山 岩的地幔源区既不是古老地幔，也不是软流圈地幔，而应是俯冲板块之上的、受到了俯冲活动 改造的上地幔贸,它的同位素组成不均一性应与俯冲作用直接有关.

南设得兰群岛地区的上地幔楔普遍受到俯冲板块来源流体的交代作用 ${ }^{[7]}$. 鉴于现残存 于南设得兰海沟的俯冲洋壳相当年轻 (14Ma) $)^{[21]}$, 以及由图 2 显示的乔治王岛第三纪火山岩 未明显受到蚀变洋壳影响, 推测第三纪俯冲洋壳岩石较新鲜。另外,乔治王禹晚白严世火山岩 的同位素组成 $\left(I_{\mathrm{Sr}}=0.703139 \sim 0.703148, \varepsilon_{\mathrm{Nd}}(t)=6.04 \sim 6.66\right)^{[22]}$ 表明第三纪以前的地幔 为亏损地幔. 这种亏损地幔如果仅受到未蚀变洋壳脱水形成的流体的交代, 虽也可能一定程 度富集不相容元素等, 但却不会产生明显的 $\mathrm{Sr}$ 和 $\mathrm{Nd}$ 同位素组成的变化, 尤其不能明显地富 放射性成因铅. 只有将俯冲沉积物来源流体的交代作用也考虑在内, 才能对地幔源区的这种 同位素组成特征作出合理的解释.

乔治王岛第三纪火山岩矿物熔融包裹体研究表明, 其地幔源区中的流体相以 $\mathrm{CO}_{2}$ 为主, 并含一定的 $\mathrm{CO}$ 和 $\mathrm{CH}_{4}$, 而 $\mathrm{H}_{2} \mathrm{O}$ 较少 ${ }^{[23]}$, 揭示了一种还原环境. 富 $\mathrm{CO}_{2}$ 流体的最可能来源是 富含碳酸盐的深海沉积物, 因为它可由后者在俯冲过程中发生脱碳酸盐作用而产生, 并且还保 持了深海沉积物本身特有的高 $\mathrm{Th} / \mathrm{U}$ 的特征 ${ }^{[24,25\}}$. 在还原环境中, $\mathrm{Pb}$ 在流体中的活动性大 于 $\mathrm{U}^{[26]}$, 因此这种高 $\mathrm{Th} / \mathrm{U}$ 和高 $\mathrm{Pb} / \mathrm{U}$ 的富 $\mathrm{CO}_{2}$ 流体的交代作用可使地幔的 ${ }^{208} \mathrm{~Pb} /{ }^{204} \mathrm{~Pb}$ 和 ${ }^{207} \mathrm{~Pb} /{ }^{204} \mathrm{~Pb}$ 相对于 ${ }^{206} \mathrm{~Pb} /{ }^{204} \mathrm{~Pb}$ 大大提高.

致谢 本工作为国家教委博士点基金资助项目。

\section{参考文献}

1 Weaver S D, Saunders A D, Tamey J. Mesozoic-Cenozoic volcanism in the South Shetland Islands and the Antarctic Peninsula: geochemical nature and plate tectonic significance. In: Craddock $C$ ed. Antarctic Genscience, Madison: University of Wisconsin Press, 1982. 263 273

2 Smellie J L, Pankhurst R J. Thomson M R A. et al. The geology of the South Shetland Islands II. stratigraphy, geochemistry and evolution. Brit Ant Surv Sci Rept. 1984, 87: 1 83

3 Birkenmajer K, Narebski W, Bakun-Czubarow N, et al. Geochemistry and petrogenesis of calc-alkaline Mesozoic volcanics and Andean plutons of inner Admiralty Bay, King George Island (South Shetland Islands, West Antarctica). Stud Geol Pol, 1985, 81:7 51

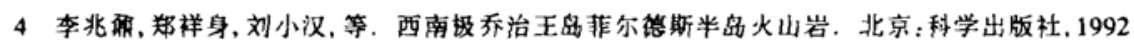

5 金灰民、匡福样、阮宏宏、等。南极菲尔德斯半禹火山作用及岩浆演化。南京: 江苏科技出版社、1992 
6 Barbieri M, Birkenmajer K, Delitala W C, et al. Preliminary petrological, geochemical and Sr isotopic investigation on the Mesozoic to Cainczoic magmatism of King George Island, South Shetland (West Antarctica). Miner Petrogr Acta, 1989, $X X X$ II : 37 $\sim 49$

7 Keller R A, Fisk M R, White W M, et al. Isotopic and trace element constraints on mixing and melting models of marginal basin volcanism, Bransfield Strait, Antarctica. Earth Planet Sci Lett, 1992, 111: 287-303

8 DePaolo D J. Neodymium Isotopic Geochemistry: An Introduction. Berlin: Springer-Verlag, 1988

9 Ellam R M, Hawkesworth C J. Elemental and isotopic variations in subduction related basalts: evidence for a three component model. Contrib Mineral Petrol, 1988, 98: $72 \sim 80$

10 Stern C R, Frey F A. Futa K, et al. Trace element and $\mathrm{Sr}, \mathrm{Nd}, \mathrm{Pb}$ and $\mathrm{O}$ isotopic composition of Pliocene and Quaternary alkali basalts of the Patagonian Plateau lavas of southernmost South America. Contrib Mineral Petrol, 1990, $104: 294 \sim 308$

11 Hegner E, Smith I E M. Isotopic compositions of the late Cenozoic volcanics from southeast Papua New Guinea: evidence for multi-component sources in arc and rift environments. Chemical Geology, 1992, 97: 233 249

12 Hart S R. A large-scale isotope anomaly in the Southern Hemisphere mantle. Nature, 1984, 309: $753 \sim 757$

13 Chase C G. Oceanic island $\mathrm{Pb}$ : two-stage histories and mantle evolution. Earth Planet Sci Lett, 1981, $52: 277 \sim 284$

14 Mukasa S B, McCabe R, Gill J B. Pb isotopic compositions of volcanic rocks in the West and East Philippine island arcs: presence of the Dupal isotopic anomaly. Earth Planet Sci Lett, 1987, 84: 153 164

15 Mahoney J J, Natland J A, White W M, et al. Isotopic and geochemical provinces of the Western Indian Ocean spreading centers. J Geophys Res, 1989, 94: 4 033 4052

16 Hart S R. Heterogeneous mantle domains: signatures, genesis and mixing chronologies. Earth Planet Sci Lett, 1988, 90: 273 296

17 Castillo P R. Origin and geodynamic implication of the Dupal isotopic anomaly in volcanic rocks from the Philippine island arcs. Geology, 1996, 24(3): 271 274

18 Milne A J, Millar 1 L. Mid-Paleozoic basement in eastern Graham Land and its relationship to East Antarctica. In: Thomson M R A, Crame J A, Thomson J W eds. Geological Evolution of Antarctica. Cambridge: Cambridge University Press. 1991. $335 \sim 339$

19 Willan R C R, Swainbank I G. Galena lead isotopic variations in a Mesozoic-Cenozoic Andean arc, Antarctic Peninsula. J Geol Soc London, 1995, 152: 767 778

20 Hole M J, Kempton P D, Millar I L. Trace-element and isotopic characteristics of small-degree melts of the asthenosphere: evidence from the alkalic basalts of the Antarctic Peninsula. Chemical Geology, 1993, 109: $51 \sim 68$

21 Maldonado A, Larter R D, Aldaya F. Forearc tectonic evolution of the South Shetland margin, Antarctic Peninsula. Tectonics, 1994, 13(6): $1345 \sim 1370$

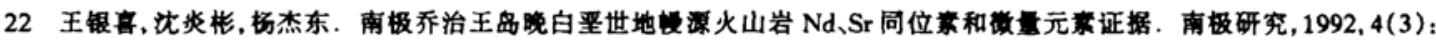
$16 \sim 30$

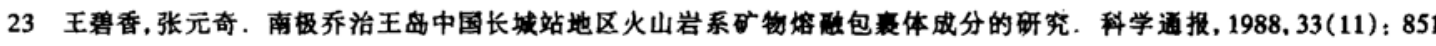
$\sim 854$

24 Bailey E H, Ragnarsdottir K V. Uranium and thorium solubilities in subduction zone fluids. Earth Planet Sci Lett, 1994, 124: $119 \sim 129$

25 Peacock S M. Fluid processes in subduction zones. Science, 1990, 248: 329 337

26 Noll Jr P D. Newsom H E, Leeman W P. The role of hydrothermal fluids in the production of subduction zone magmas: evidence from siderophile and chalcophile trace elements and boron. Geochim Cosmochim Acta, 1996, 60(4): 587 611 